\title{
An audit to analyse the two-week wait pathway at an oral cancer specialist district general hospital
}

\author{
Ariyan S. Araghi, ${ }^{* 1}$ Yasmin Harris² and Panayiotis Kyzas
}

\section{Key points}

This audit aims to elaborate on what happens once a two-week wait urgent cancer referral is made. It elaborates on the process and more specifically the timeframe from referral to initial treatment.
It brings the reader's attention to upcoming changes to the timeframe in which oral cancer patients must be seen, diagnosed and treated as per the NHS Long Term Plan (2020). It also touches on some of the barriers that exist to aid in the early detection of this disease.
It is hoped that the audit brings home the importance of carrying out a conclusive oral cancer screen, whilst ensuring the correct referral is made to secondary care.

\begin{abstract}
Background The incidence of head and neck cancers is increasing, alongside a decrease in associated mortality. Currently, medical and dental practitioners can refer patients to be seen urgently within two weeks. The appropriateness of these referrals has been disputed. In 2020, the Department of Health aims for patients to be given cancer diagnoses within 28 days from referral.
\end{abstract}

Methods A retrospective audit was conducted for all patients referred under the two-week wait pathway in a six-month period. In the first cycle of this audit, one month's worth of urgent referrals were analysed; given the small sample size, very few recommendations could be made. The audit cycle was repeated and it analysed six months' worth of data, which gave a much more representative study. All patients were analysed to see if the 14-day period had been breached. Positive cancer patients were further assessed to see if their diagnosis had been given within 28 days and treatments within 62 days.

Results Of the 569 patients seen, there was a positive malignancy diagnostic yield of $7.38 \%$. Nineteen patients breached the 14 -day wait. Of the positive patients, $45.2 \%$ received their diagnosis more than 28 days from referral, and $22.2 \%$ of these patients received treatment after 62 days.

Conclusion The department performed well despite the high number of referrals. This audit has touched on some key issues which have been discussed in detail in this article. Furthermore, this audit recommends a concerted effort to improve oral cancer detections skills among GDPs and GMPs. While all referrals may be appropriate from a primary care point of view, this audit makes it apparent that better differentiation is needed between malignant and routinely manageable lesions. All secondary care units alongside general practitioners can learn from the findings of this audit.

\section{Preface}

The incidence of head and neck cancers (HNCs) in the UK is rising. The necessity of early and appropriate recognition of patients at risk is incredibly high. The National Institute of Health and Care Excellence (NICE) have released guidance for the recognition of symptoms that warrant further investigation. Cross-pathway referral for these cancers allows both general medical practitioners (GMPs)

${ }^{1}$ North Manchester General Hospital, Delaunays Road, Crumpsall, Manchester, M8 5RB, UK; ${ }^{2}$ Manchester Medical Society, University of Manchester, Manchester, M13 9PL, UK. ${ }^{*}$ Correspondence to: Ariyan Araghi

Email address: ariyanaraghi@live.co.uk

Refereed Paper.

Accepted 12 February 2020

https://doi.org/10.1038/s41415-020-1449-2 and general dental practitioners (GDPs) to refer patients to be seen within two weeks by a specialist secondary care consultant in an oral maxillofacial cancer unit. Despite this, the positive diagnosis percentages of these cancers after referral remains very low. This low percentage is a promising variable on a patient level; however, it highlights an area of education for the wider team. Additionally, when compared with neighbouring European countries, the mortality one year post-diagnosis is lower in the UK. This calls into question the appropriateness of the urgent two-week wait $(2 w w)$ referral pathway for these cancers and its efficiency in triaging patients from primary care to secondary care.

As part of the NHS Long Term Plan, the Department of Health have decided to implement a new target for potential cancer patients, which aims to intercept and treat cancer sooner. In 2020, all cancer diagnoses should be given within 28 days from the initial referral, thus shifting the importance from date of secondary care consultation to date of diagnosis. The details of the NHS Long Term Plan are touched on in more detail later on in this article.

\section{Aims}

This project is a retrospective audit of all the patients referred to the head and neck team at North Manchester General Hospital under the urgent $2 \mathrm{ww}$ referral for suspected HNCs in a six-month period. This study has the following aims:

1. To assess whether all of the patients referred are being seen in the required 14 days, according to the current ' $2 w w$ ' guidelines 


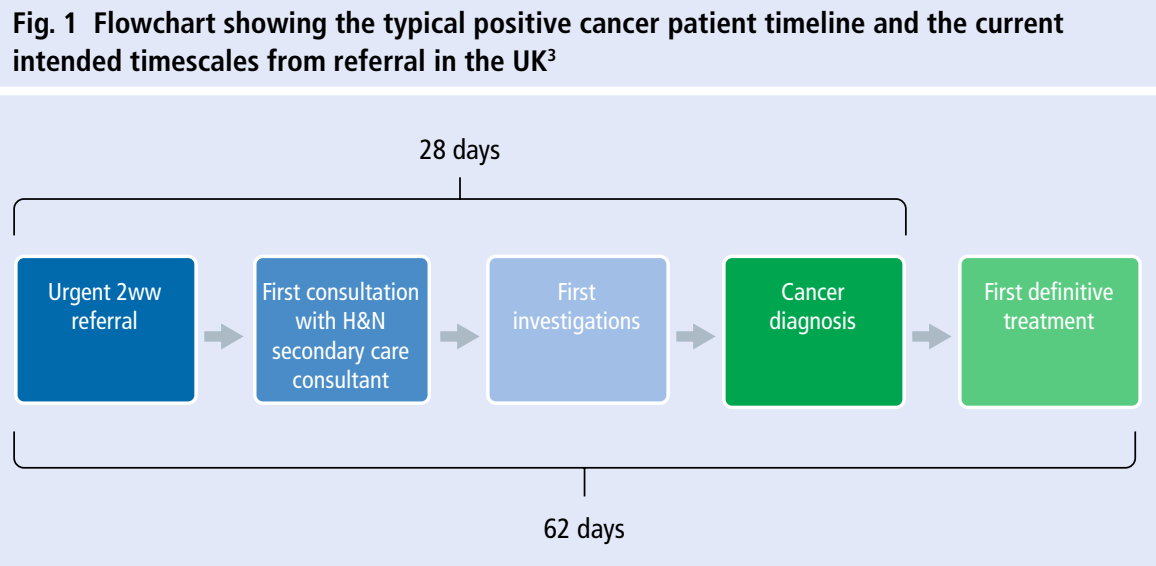

2. To assess the positive diagnostic yield of cancer in this group of patients

3. Of the positive cancer patients, to assess whether they are being given treatment within 62 days and ascertain what treatment is being given

4. To analyse whether the positively diagnosed cancer patients were being given their initial diagnosis within 28 days, in preparation for the new guidelines to be implemented in the NHS Long Term Plan (2020)

5. To help develop guidance in which a nationalised plan can be utilised to ensure maximum treatment efficacy for urgent cancer referrals.

The standard set for this audit would be that $100 \%$ of the patients were being seen initially in 14 days. One hundred percent of the patients positively diagnosed with cancer would receive diagnosis within 28 days and subsequent treatment within 62 days in line with the current guidelines for the $2 \mathrm{ww}$ referral system for HNCs. The standard for diagnostic cancer yield/positive oncological transformation would be set at $3 \%$, as appropriate for a screening test as set out by NHS England.

\section{Background}

In the UK, the incidence of HNCs has doubled since 2006 and 2010, with the number of new diagnoses continuing to increase. ${ }^{1} \mathrm{HNCs}$ in general have five-year survival rates of $55 \%$ in the UK. ${ }^{2}$ The outcomes are known to be worse the more advanced the disease is picked up.

In an attempt to ensure that $\mathrm{HNC}$ patients are being seen and treated efficiently, in 2000, the Department of Health (DOH) released guidance that should be adhered to when referring patients with an urgent suspicion of cancer. This is termed the $2 \mathrm{ww}$ pathway. ${ }^{3}$ The typical journey for a patient positive for $\mathrm{HNC}$ is highlighted in Figure 1. As shown, it aims for patients referred under the $2 \mathrm{ww}$ pathway to be seen by a secondary care specialist consultant within 14 days. The patient's first definitive treatment (FDT) - that is, surgery and chemotherapy - should be within 62 days. ${ }^{3}$

HNCs can vary widely in their presenting symptoms. In order to identify patients at risk, the NICE published a set of guidelines (NG12) highlighting symptoms that warrant a $2 \mathrm{ww}$ referral. ${ }^{4}$ Both GMPs and GDPs can choose to refer patients with the following symptoms: 1. Laryngeal cancer: persistent unexplained hoarseness or unexplained lump in the neck in patients aged over 45

2. Oropharyngeal cancer: unexplained ulceration in the oral cavity for over three weeks or a persistent and unexplained lump in the neck

3. Thyroid cancer: an unexplained thyroid lump. ${ }^{4}$

The guidelines were reviewed and updated in 2017 for GMPs to refer to GDPs for a second opinion, rather than direct $2 \mathrm{ww}$ referral, in the following cases:

- A lump on the lip or oral cavity

- A red, or red and white, patch in the oral cavity consistent with erythroplakia or erythroleukoplakia. ${ }^{4}$

The update also removed the requirement for the oral cavity patch to be painful, swollen or bleeding. This increases the number of patients that would be 'appropriate' to refer. ${ }^{5}$

The aim of the $2 \mathrm{ww}$ referral system is to be a screening tool for malignant disease. Therefore, it can be reasonable to expect a $10 \%$ positive oncological transformation/malignant diagnostic yield. However, when developing the guidelines for referral, it is important to also take into account departmental resources and balance this with a potential large amount of patients that are deemed to be appropriate to refer.

Langton et al. conducted a systematic review of 17 studies showing all patients referred from 2000 to 2014 under the $2 \mathrm{ww}$ for HNCs. The review showed that the proportion of patients diagnosed under the pathway was decreasing and that the positive diagnostic yield of the $2 \mathrm{ww}$ pathway remained low between all studies. His review showed that there has been an increase in the number of referrals, without an increase in the number of diagnoses, which can potentially put immense burden on already busy secondary care departments. Langton's study further demonstrated a $60 \%$ increase in referrals between 2001-2004, which resulted in departments being unable to see patients within the two-week timeframe. ${ }^{6}$ In terms of patient experience, the $2 \mathrm{ww}$ was very beneficial as patients felt that they were being seen quickly and thus felt more in control of their diagnosis. ${ }^{6}$

More recent audits from other major head and neck centres have added to these findings. A recent audit from a head and neck cancer unit in Glasgow showed that, in one year, 7.6\% of patients referred under the $2 \mathrm{ww}$ pathway were positive for a primary HNC. ${ }^{7}$ Pindolia et al. showed that $96 \%$ of the $2 \mathrm{ww}$ referrals in a major London head and neck centre were nonmalignant, instead resulting in diagnoses such as oral lichen planus and recurrent aphthous ulceration ( $47 \%$ and $14 \%$, respectively). ${ }^{8}$ This questions whether the $2 \mathrm{ww}$ pathway is being overused by referring practitioners. Roy et al. questioned whether the increase in referral numbers could be due to the fact that not many practitioners will see this type of cancer or have had the education, training or exposure to it; therefore, they will be more likely to refer for any symptoms suggestive of HNC. Their research further highlighted that some referrals had been made without consulting the NICE guidelines at all. ${ }^{9}$

On the subject of guidelines, one could argue that the current NICE guidelines require further re-evaluation. Tikka et al. analysed the presenting symptoms of patients with positive cancer diagnoses and compared these with the NICE referral criteria. Statistical models identified nine symptoms highly linked with HNC, only four of which are in the criteria. ${ }^{10}$ Research has been done to introduce a web-based prediction score for patients with 
suspected HNCs. This would help practitioners to identify at-risk patients, concentrating on risk factors and symptoms, generating a risk score. ${ }^{11}$

Due to the cross-pathway referral system, there could be a difference in the group of referring practitioners, suggesting that a certain group requires more education in terms of referral. Shanks et al. showed that $73 \%$ of medical students asked had not been taught how to examine the oral cavity. ${ }^{12}$ In comparison, there have been highly detailed examination guidelines released for GDPs in dental journals for the positive identification of oral cancers. ${ }^{13}$ However, when reviewing audit results, there seems to be discrepancies. Hong et al. demonstrated 148 referrals from GMPs and $9.5 \%$ positive malignancy pick-up, compared with 72 referrals with $1.4 \%$ pick up from GDPs. ${ }^{14}$ Roy et al. also showed that 55\% of their referrals were from GMPs. ${ }^{9}$ In contrast, Pindolia et al. showed the majority of referrals to their HNC centre to be from GDPs. ${ }^{8}$ There is lots of conflicting and somewhat convoluted research in this area; thus, it would be safe to say a head and neck detection toolkit would be most useful for both GDPs and GMPs.

Though the $2 \mathrm{ww}$ is a very well-established target nationally, the UK government has decided to implement a new set of guidelines in the treatment of cancers. In the NHS Long Term Plan published in January 2019, a 'faster diagnosis' guideline was introduced. ${ }^{15}$ The changes to patient journey are highlighted in Figure 2. The main change will be the focus on giving patients their cancer diagnosis within 28 days of the urgent suspected cancer referral. This is to be implemented in all sites by $2020 .{ }^{15}$

This change is stated to aim to improve patients' quality of life, decreasing the time in which they are feeling anxious and starting the required treatments as soon as possible. The 62-day timeframe for FDT is to remain in place. ${ }^{15}$

\section{Methods}

PubMed and Medline database searches were used to assess what literature already exists around this subject area. The following terms were used in the search: 'head and neck cancer', '2 week wait', '2-week referral', 'UK', 'fourteen day', 'oral cancer,' 'pharyngeal cancer', 'GP referral', 'maxillofacial referral', 'oral cancer diagnosis' and 'oral cancer classification. The NICE website was used to access the most recent guidelines for the

Fig. 2 Flowchart showing the typical positive cancer patient timeline and the new change to be implemented from 2020 in accordance to the NHS Long Term Plan ${ }^{15}$

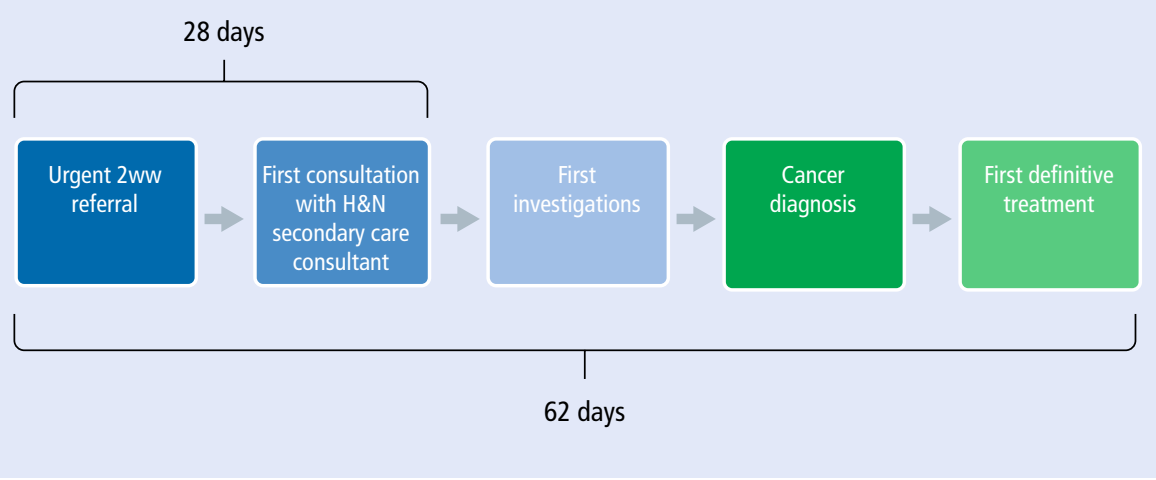

urgent referrals. The NHS England website provides the documentation with the most recent cancer waiting times and the NHS Long Term Plan.

The patient inclusion criteria were all patients referred under the $2 \mathrm{ww}$ pathway for urgent suspected HNC to North Manchester General Hospital in the six-month period between September 2018 and the end of February 2019. Patient data was obtained through the hospital data collection team; there was no need for ethical approval as patient outcomes were not affected in this retrospective audit. Local governance standards were met after a formal request was made around the audit subject area. The data provided from the hospital database outlined the below seven parameters - these parameters allowed us to conduct the audit, and compare and contrast the journey of patients from referral to consultation and beyond.

Parameters to be assessed were:

1. Date of referral

2. Date of first consultation with secondary care team

3. First investigation (if applicable)

4. Diagnosis

5. Date of cancer diagnosis

6. Date of FDT

7. Treatment type.

As alluded to earlier on in this article, the journeys of one month's worth of patients who were referred on the $2 \mathrm{ww}$ rule were analysed; the sample size was too small to give a representative conclusion as to what recommendations could be made going forward. The second round of data collection incorporated six times as much data and, as a result, several conclusions were made. Patients fell into one of two groups during the data collection: those who were taken off the cancer pathway and only matched parameters $1-4$, and a second group who remained on the cancer pathway and went on to match parameters 4-7 above. The data analysis was carried out by a junior member of the surgical team and presented at a local departmental governance meeting within the specialist cancer hospital.

The data collection process was not able to differentiate between type and severity of oral cancer - that is, we will not be able to tell the difference between a T1N0M0 (small tumour) and a T4N1M1 (large tumour with metastatic disease). This audit did not aim to distinguish between different cancer types, but rather to solely assess the timeframe in which patients are seen with regards to the $2 \mathrm{ww}$ pathway. It is unlikely that type and size of tumour should play a part in the time pathway of the diagnostic oral cancer journey; however, this audit has highlighted that this could be a variable in the journey, therefore signposting an area of potential review in the future.

\section{Results}

When both rounds of data collection were completed, analysing data from August 2018 and then in the second cycle from September 2018 to March 2019, 603 patients were referred to this centre under the $2 \mathrm{ww}$ pathway. Thirty-four patients did not attend their appointments and therefore were erased from the data to be analysed. Therefore, 569 patients were seen in the head and neck department for urgent suspected cancer.

The data showed that 19 patients of the patients seen in the department breached the $2 \mathrm{ww}(3.34 \%)$. All of these were due to patient choice except for one case, which was the sole case due to departmental capacity issues. This patient was seen in 23 days and the ultimate 
diagnosis was non-cancer. The average time to being seen for all of the patients seen was 8.5 days (standard deviation of 4.3 ).

When comparing the ultimate diagnoses with the time of meeting, one of the positive

Fig. 3 A histogram showing the frequency of each initial investigation ordered

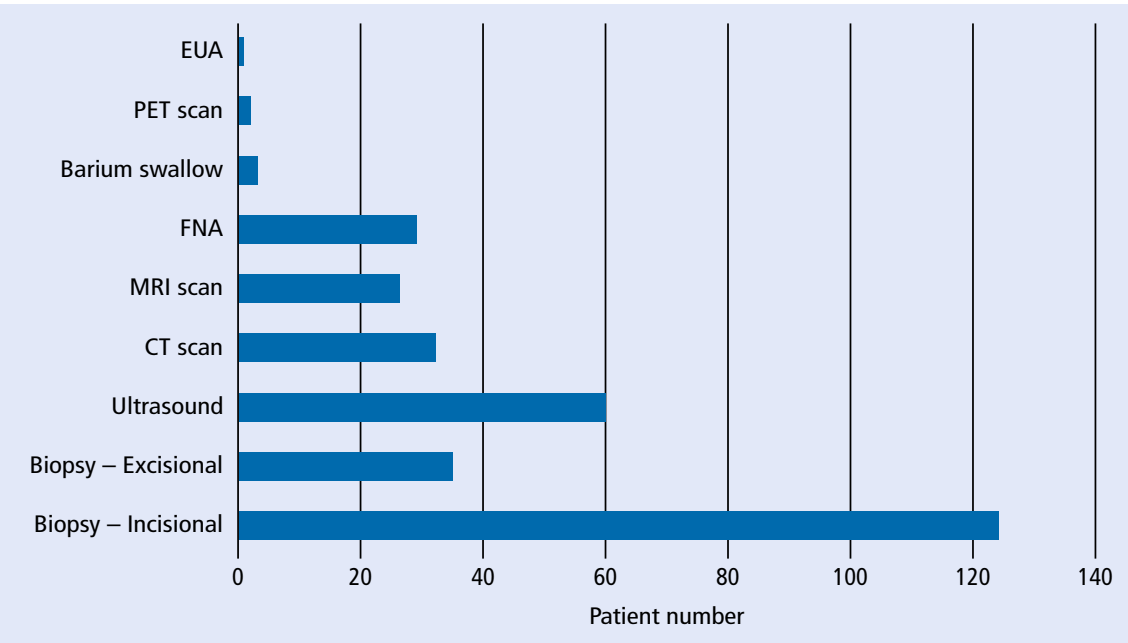

Fig. 4 A histogram showing the distribution of patients, grouped into different timings

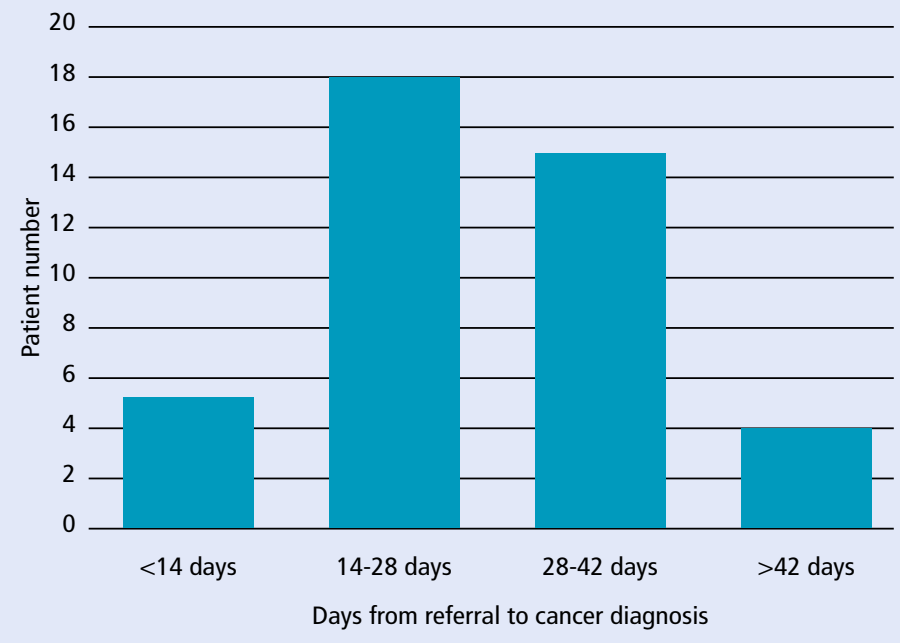

Fig. 5 Box plot analysing the skew of days between referral and cancer diagnosis

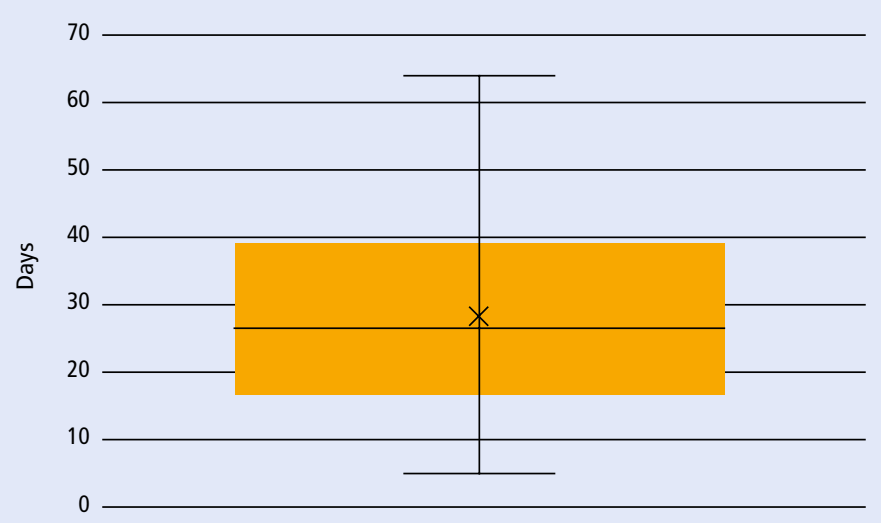

Three hundred and twelve patients (54.8\%) were sent for one of the following:

- Biopsy (incisional or excisional)

- Ultrasound

- MRI scan

- Fine needle aspiration

- CT scan

- Barium swallow test

- Examination under anaesthesia (EUA)

- PET scan.

Forty-two out of 603 patients were positively diagnosed with cancer $(7.38 \%$ malignancy yield) in the data analysed.

Figure 3 shows the frequency of each diagnostic investigation. Three of the 42 positive cancer patients (see Fig. 3) had no initial investigations. Of the $32 \mathrm{CT}$ scans requested, 16 were positive for cancer (the highest diagnostic yield of 50\%). Despite being the most requested investigation, the incisional biopsies were positive for cancer in 11 cases (8.9\%).

Analysis of the date of diagnosis showed that 19 of the 42 positively diagnosed patients (45.2\%) had been diagnosed after the target of 28 days. The distribution of timings for each group of dates can be seen in Figure 4.

The mean time between referral and diagnosis was 28.4 days, with standard deviation of 13.6. The most that a patient waited for a diagnosis was 64 days, seen in Figure 5.

Five patients were not given a definitive treatment and one patient was awaiting surgery, which left 36 of the 42 cancer patients with treatment. Eight patients breached the 62-day period (22.2\%), and the results of each group can be seen in Figure 6 .

Comparing the FDT date with the date of diagnosis revealed that five of the eight patients who had passed the 62-day FDT target had also failed to reach the 28 day target for diagnosis. Figure 7 shows the distribution of treatment types given to these patients and their frequencies.

\section{Discussion}

The unit received referrals for over 100 patients per month. It was interesting to note the 34 patients who did not attend their consultations for suspected cancer. This could be tackled by increasing communication in primary care about the importance of these consultations and explanation that this could be a potentially malignant disease. Before this, practitioners 
should be well-informed about what is definitely oral cancer and how the lesions present.

The data has shown that the unit is very familiar with the 14-day wait and has been able to deal with the large number of patients well, with only one breach in all 569 patients being due to lack of departmental space. Further investigation is required into the exact reason for this delay, which resulted in a 23-day wait. The team are able to meet well-known targets and also accommodate the 18 patients that need to delay their consultations. The average waiting time is very short. The longest that a patient had to wait for their initial consultation was 34 days, again due to personal choice.

The one patient that had breached the 14-day period with an ultimate cancer diagnosis is the same patient that had a 117-day delay between referral and FDT (Fig. 7). This patient had also been diagnosed at 41 days after referral (Fig. 5). This questions whether there had been some special circumstances in this one patient which required certain delays. This would be something that could benefit from further case-specific investigation.

The patients were being investigated appropriately, with the majority of patients sent for initial diagnostic investigations. Figure 3 shows that 159 patients altogether were subjected to biopsies. The fact that the diagnostic yield for patients sent for CT scans was so high means that the consultants are appropriately sending patients for the correct investigations. This shows us that more worrying patients are sent for CT scans as they are more likely to be malignant after initial clinical history and examination. Incisional biopsies are ordered when histology is required for the diagnosis, resulting in a lower yield.

The malignancy yield from this cohort of patients was low at $7.6 \%$. This is in keeping with the audits and reviews from other sites, further questioning the $2 \mathrm{ww}$ pathway symptom guidelines. ${ }^{7,8,9}$ There is no dispute in the necessity of patients being seen promptly for suspected HNCs. However, this does highlight the high sensitivity and low specificity of the $2 w w$ as a screening tool. Many patients are being seen, resulting in more diagnoses. This is very positive, but only because the unit have proven to be prepared to handle such a large number of patients.

Is there a way to decrease the number of patients being seen, while keeping the high sensitivity of positive diagnoses? The question lies with the referring practitioners.

\section{Fig. 6 Histogram showing the groupings of patients according to how many days from referral to FDT}

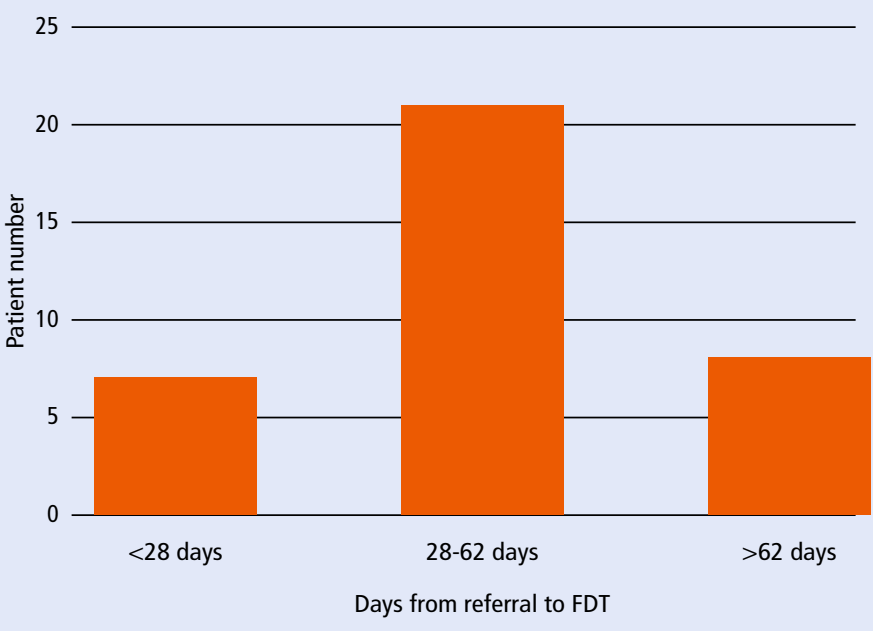

Fig. 7 Pie chart showing the frequency of each different treatment type

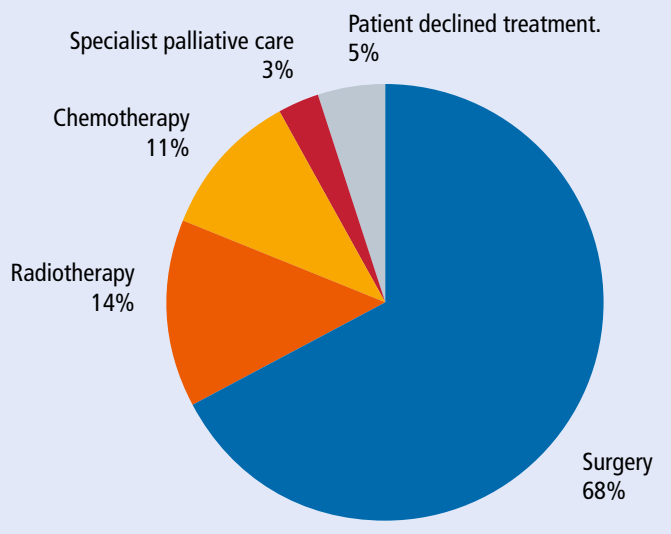

As previous research has shown, there is a possibility of bringing in a more detailed online referral system. ${ }^{11}$ Research has shown that improved communication between the referring practitioner and the secondary care consultant could stop patients that are not at risk from being seen. ${ }^{14}$ A preliminary suggestion is that an online toolkit should be developed to help practitioners definitively diagnose high-risk lesions, as the current system is proving inefficient in this sense. There are some toolkits that are available online, these are sign posted later on in the discussion; going forward from this, every effort should be made to raise awareness of these educational tools.

Ultimately, these methods both rely on improving the education of the referring professional. It is possible that adequate training is not being given to GMPs and GDPs for the referral of oral lesions. As mentioned in previous studies, referring practitioners may have a low tolerance for referral under the $2 \mathrm{ww}$ due to the worry of missing malignant conditions that they are not familiar with, and the potential repercussions to both patient health and their own professional record. ${ }^{9}$

It does state in the NICE guidelines that referrals for oral lesions can be done via a second opinion with a GDP. ${ }^{4}$ However, the literature states that the majority of referrals were via GMPs, this brings into question whether the cross disciplinary protocol is being adhered to or not. ${ }^{9,14}$ In hindsight, it would have been useful to compare the numbers of referrals and subsequent malignancy yield of referrals from GMPs and GDPs - this could be an area that we visit in a future study.

From the work conducted by Shanks et al., it is evident that further education in terms of oral cavity examination is required for medical students. ${ }^{12}$ It seems as though the 
medical curriculum has overlooked this topic for undergraduate medical students. ${ }^{13}$ Undergraduate educators should consider the importance of overlooking this area of teaching and incorporate essential oral pathology/oncology into the undergraduate curriculum.

The target for having a cancer diagnosis within 28 days from referral was poorly met, being achieved in only $54.8 \%$ of cases. This is far off the $100 \%$ set standard. Figure 4 shows us that the majority of patients that were seen after this target were at least diagnosed within 42 days ( 15 patients), and in four cases this was over 42 days. Figure 5 shows us that even the mean time for diagnosis was 28.4 days, greater than the target.

It is important to realise that, according to the previous guidelines, all of these patients (apart from the one that has been mentioned previously) had reached the 14-day referral target. However, they still had been given a fairly late diagnosis, thus failing to reach the new cancer target for the NHS Long Term Plan (2020). Departmental and institutional understanding of this target must be assessed.

Diagnosis date mainly relies on two things: the administrative team being able to book in and accommodate patients for follow-up appointments, alongside the radiology and histopathology teams being able to conduct relevant investigations and report on these promptly. The department have been able to do this for the majority of patients, however it has proved increasingly difficult as the number of urgent referrals are very high. Units are now pooling services and joining forces with other hospitals to help share the workload and ensure patients receive timely interventions. For example, several hospital units have joined forces in the north west and review all HNCs together. This shared workload, increased availability of resources and greater secondary care expertise is ensuring that patients are receiving the correct intervention within the right time frame, this is now known as the 'pan hospital MDT scheme'. Another recommendation is for the oncology leads in similar units to put together a focus group, ensuring that, nationwide, all departments will have the know-how and ability to be compliant with the NHS Long Term Plan.

The statistics for FDT dates were positive; however, as only $77.7 \%$ of patients were being treated within 62 days, the standard set of $100 \%$ was not met. The distribution seen in Figure 6 shows us that the department were able to treat seven patients very rapidly, with FDT being below 28 days. These patients had been treated with surgery in five cases, active monitoring and palliative care. Therefore, it is possible for procedures to be scheduled. Essential to note is that all of these patients had been given a diagnosis in less than 28 days.

The comparison between diagnosis date and FDT has shown a link, as five of the eight breaches of 62-day FDT had also breached the 28-day diagnosis. However, this is not necessarily causative, as of the 19 cases that breached the 28-diagnosis target, only five had later treatments. This does further support the NHS Long Term Plan (2020), with earlier diagnosis hopefully leading to earlier effective treatment of these patients.

Further investigation regarding the two outlier patients with waiting times over 100 days would be required (Fig. 7). It is possible that, as the FDT for both of these patients was surgery, the wait could be due to the availability of high-dependency unit beds or other common causes of operation delays. The other cases that breached 62 days were due to chemoradiotherapy centre waiting times and active monitoring. Looking at Figure 7, the proportion of FDTs is unsurprising, with the vast majority of cases resulting in the need for surgery.

\section{Conclusion}

This project has highlighted that the Head and Neck Unit at North Manchester General Hospital is meeting the $2 \mathrm{ww}$ target well, despite having a very large cohort of patients. The low malignancy diagnostic yield from this retrospective audit has added to previous centre audits in questioning the appropriateness of using the NICE referral symptoms alone for identification of HNCs. There needs to be further education for referring primary care practitioners, both GMPs and GDPs. This has already been tackled in part by the development of a new model for simplifying the management of oral cancer patients. We would encourage the reader to refer to the following resource: 'The reconstructive oral cancer patient: what the general dental practitioner needs to know' (2019), ${ }^{16}$ and the plethora of information it posts the reader to in aiding with proper oral cancer care and diagnosis.

Although patients were mainly receiving their FDTs in adequate time, a large proportion were not meeting the 28-day diagnosis timeframe. Hopefully, with the awareness generated from presentation of these results, this will be improved in preparation for the upcoming NHS Long Term Plan.

In the current pandemic that we face, we have been trialling a new method for seeing HNC $2 \mathrm{ww}$ patients. We are requesting that a photo is sent in with any suspicious lesion alongside the referral, this allows us to expedite or delay clinic appointments according to the what the photos shows us. The limitation of this is that is does not account for non visible signs of HNC such as dysphonia, dysphagia or otalgia. However, our experience to date has showed us that this reduces hospital foot flow and highlights the patients who definitively require investigation. Once this subgroup arrive, we have created a one step clinic where a consultation, exam and possibly biopsy will be performed in the same visit. The aim of this is to again reduce hospital visits for patients, moreover it ensures that the next visit will entail diagnosis and initial treatment planning. This new modality shows us that we are well in line with the NHS 2020 Long Term Plan.

It is hoped that the reader realises that the onus for correct management and succinct diagnosis relies on the original referrer. The World Health Organisation (WHO) has long battled for a universal screening programme for oral cancer detection; this outcry has yet to prove successful. ${ }^{17}$ The UK National Screening Committee have a 20 -step checklist before allowing nationwide enrolment of a screening programme; multiple suggested oral cancer screens have all failed to meet the required number of criteria to allow progression into experimental trials and eventual nationwide screening. We would encourage the reader to familiarise themselves with the work of Brocklehurst et al., ${ }^{17}$ who review the advantages and disadvantages of oral cancer screening, analysing the complex health economics of the matter.

The following recommendations have been summarised as a result of this audit and it is hoped that all members of the wider healthcare team can gain from the conclusions below:

- To try and develop a focus group to create guidance for the UK-wide clinicians regarding the 2020 NHS initiative

- To develop a series of educational lectures for undergraduate medical students to inform them about basic oral pathology and oncology. This could be done by adding to the undergraduate medical curriculum 
- An online toolkit should be developed to help ensure that the correct lesions are being sent in on the $2 \mathrm{ww}$ system. The available systems by Cancer Research UK and the BDA should be expanded on and publicised more

- Ultimately, this audit has shown that the $2 \mathrm{ww}$ urgent referral system is being overused. One would hope that the readers of this audit realise the importance of updating their knowledge in oral cancer detection and diagnosis. This will have a positive impact on a multidimensional basis; patients have the best chance of early detection and definitive cure, the referral system can be used to triage suspicious lesions only, and non-suspicious lesions can be managed locally, thus removing strain from the already convoluted hospital system

- This audit will be repeated in the coming years after the NHS Long Term Plan has been fully implemented; it would be useful to see if the aforementioned analysis and suggestions will have a positive impact on first treatment dates.

\section{References}

1. Mehanna H, Evans M, Beasley M et al. Oropharyngeal cancer: United Kingdom National Multidisciplinary Guidelines. J Laryngol Otol 2016; 130: 90-96.

2. Oxford Cancer Intelligence Unit. Profile of Head and Neck Cancers in England: Incidence, Mortality and Survival. 2010. Available at http://www.ncin.org.uk/view?rid=69 (accessed March 2019).

3. NHS England. Waiting Times for Suspected and Diagnosed Cancer Patients: 2016-17 Annual Report. 2017. Available at https://www.england.nhs.uk/statistics/wp-content/ uploads/sites/2/2017/06/Cancer-Waiting-Times-AnnualReport-201617-1.pdf (accessed March 2019).

4. National Institute for Health and Care Excellence. Referral guidelines for suspected cancer. 2011. Available at https:// www.nice.org.uk/guidance/cg27/documents/cg27-referralfor-suspected-cancer-review-decision2 (accessed March 2020)

5. Grimes D, Patel J, Avery C. New NICE referral guidance for oral cancer: does it risk delay in diagnosis? Br J Oral Maxillofac Surg 2017; 55: 404-406.

6. Langton S, Siau D, Bankhead C. Two-week rule in head and neck cancer 2000-14: a systematic review. Br J Oral Maxillofac Surg 2016; 54: 120-131.

7. Douglas C M, Carswell V, Montgomery J. Outcomes of urgent suspicion of head and neck cancer referrals in Glasgow. RCS Annals 2019; 101: 103-106.
8. Pindolia $H$, Berridge $N$, Perry M. A review of the utilisation of the 2weekwait referrals in a large Maxillofacial unit in London. Br J Oral Maxillofac Surg 2018; DOI: 10.1016/j. bjoms.2018.10.025.

9. Roy S, Anjum K. The two-week wait-a qualitative analysis of suspected head and neck cancer referrals. Br Dent J 2018; 225: 159-163.

10. Tikka T, Pracy P, Paleri V. Refining the head and neck cancer referral guidelines: a two centre analysis of 4715 referrals. Br J Oral Maxillofac Surg 2016; 54: 141-150.

11. Lau K, Wilkinson J, Moorthy R. A web-based prediction score for head and neck cancer referrals. Clin Otolaryngol 2018; 43: 1043-1049.

12. Shanks L A, Walker T W, McCann P J, Kerim M J. Oral cavity examination: beyond the core curriculum? Br J Oral Maxillofac Surg 2011; 49: 640-642.

13. Lewis M A O. Mouth cancer: presentation, detection and referral in primary dental care. Br Dent J 2018; 225: 833-840.

14. Hong B, Shaikh Z, Adcock S, Aldallal S N. Two-week wait false alarms? A prospective investigation of $2 \mathrm{ww}$ head and neck cancer referrals. Br Dent J 2016; 220: 521-526.

15. NHS England. The NHS Long Term Plan. 2019. Available at https://www.longtermplan.nhs.uk/wp-content/ uploads/2019/08/nhs-long-term-plan-version-1.2.pdf (accessed March 2019).

16. Araghi A, Nasry H. The reconstructive oral cancer patient: what the general dental practitioner needs to know. $\mathrm{Br}$ Dent J 2019; 227: 689-692.

17. Brocklehurst $P$, Speight $P$. Screening for mouth cancer: the pros and cons of a national programme. Br Dent J 2018; 225: 815-819. 